\title{
Public Health Optimism in the New Millennium
}

\author{
Yemane Berhane $^{1}$
}

The hope for prosperity in the new Ethiopian millennium is very high. Celebrating a millennium is a rare event and it is indeed a historic moment for every body to wish for better life. Ensuring reasonable standards of health is the basis for prosperity, for individuals and the nation. In Ethiopia modern health services started expanding about hundred years ago. Although some remarkable achievements have been observed in the recent decades there are still formidable challenges for creating healthy populations and environment.

The Ethiopian population grew by about sevenfold in the last century. Migration and urbanization created a serious challenge in preventing and controlling communicable diseases due to low immunity among migrant populations and overcrowding and poor sanitation in urban areas. Environmental degradations resulted in irregular climatic conditions leading to frequent drought and famine. Considerable proportion of the Ethiopian population lives under poverty level. Malnutrition became rampant among children and mothers maintaining a vicious cycle of malnutrition, infection and poverty. Morbidity and mortality rates have been on of the highest in the world.

In Ethiopia, health services have been poorly organized and their distribution has been strongly biased towards urban areas. Although health services have been greatly expanded in the last two decades the shortage of the necessary human and material resources seriously curtailed expansion of reasonable quality services to the neediest segments of the population. Cultivating the right attitude and skills among providers has not been achieved to the desired level. Consumers of health services remain largely unsatisfied with the services being rendered. The population has not yet internalized the culture of prevention and control of health problems and early utilization of health care as appropriate and when needed. Economic, social, cultural and geographic barriers to health services remain serious challenges. Uptake of health services such as immunization and family planning remain much lower than desired despite enormous efforts by the governments of Ethiopia and external stakeholders.

The occurrence of new and deadly communicable diseases such as HIV/AIDS and the increasing occurrence of non communicable diseases (NCD) overstretched the limits of the health sector. In addition, the loss of health professional due to these health problems and brain drain has compounded the problem.

Efforts to train different categories of health professionals have been intensified in the last two-three decades; especially in the last decade. However, that was paralleled by the increasing level of brain drain. In addition, the sudden increase in quantity of production has made maintaining quality a serious challenge for training institutions. In recent years "brain shifting" added a new dimension to the human resource crisis. New actors in the health sector inadvertently distort the human resource capacity building process by pulling out trained health workers from their main professional duties to subsidiary tasks.

Health professional associations have been flourishing over the last two-three decades. These associations have been instrumental in some key advocacy works and mobilizing the health professionals for a good cause of improving professional standards. However much remain to be done by professional associations in terms pf promoting better professional standards and in demanding better health for populations.

The way forward needs to be much focused if success in improving the health status of our population is seriously desired. Much coordinated and collaborative efforts are needed more than ever now in order to achieve success. There is very little room for solitary 
actions; all stakeholders in health and development need to communicate well and act together. Only large scale and coordinated efforts can overcome the formidable challenges in achieving better health.

The Government ought to play the central and coordinating role in mobilizing resources and engaging every one for common goals and visions. Health programs shall use all available resources with well defined long term goals. Reliance on the external resources ought to be limited to the absolute necessity. The Government must also respond appropriately to the needs of the health professionals. The community needs to be mobilized appropriately to take responsibility for its health.

Health training institutions need to be given adequate resources and motivations to produce the much needed health professionals in adequate number and acceptable quality. They need adequate autonomy to be innovative and proactive in the health sector. Training schools also need to strive for better standing to be able to produce the necessary number of health cadres with the required skills.

Health care providers, whether working in the public or private sector, need to be responsible for ensuring reasonable quality for the services they are rendering. The safety of health service consumers must be considered first in all their actions. Appropriate considerations must be given in providing services to the disadvantaged and most vulnerable segments of the population.
Professional associations must develop visions that help achieve better health for the Ethiopian population. They need to be proactive and take leads to mobilize the community and the professionals around their goals and visions. They need to strive for better professional standards.

External friends of Ethiopia must be more responsive to the needs of the nation and work in partnership with all stakeholders in harmony. They must take appropriate precaution to avoid facilitating fragmentation and demobilization of the work force and in all their good efforts investing in human capacity building must be considered.

In conclusion, the Ethiopian people deserve to be healthier in the new millennium. All stakeholders must act responsibly and in unity to make the dream a reality. This very brief discourse will hopefully inspire professional and health policy makers to engage in more productive deliberations to find to overcome challenges and succeed in their endeavors in making health a development asset.

\section{Reference}

Berhane Y, Hailemariam D, Kloos H (Eds): The Epidemiology and Ecology of Health and Disease in Ethiopia. Shama Books, Addis Ababa, Ethiopia. 2006 